EPJ Web of Conferences 59, 17002 (2013)

DOI: $10.1051 /$ epjconf/20135917002

(C) Owned by the authors, published by EDP Sciences, 2013

\title{
Dynamics of the spectral behaviour of an ultrashort laser pulse in an argon-gas-filled capillary discharge-preformed plasma channel
}

\author{
S. Sakai ${ }^{1}$, T. Higashiguchi ${ }^{1,2, a}$, N. Yugami ${ }^{1,2}$, N. Bobrova ${ }^{3}$, Y. Sentoku ${ }^{4}$ \\ and R. Kodama ${ }^{2,5}$ \\ 1 Utsunomiya University, Department of Advanced Interdisciplinary Sciences, Yoto 7-1-2, \\ Utsunomiya, Japan \\ 2 Japan Science and Technology Agency, CREST, 4-1-8 Honcho, Kanagawa, Japan \\ 3 Institute for Theoretical and Experimental Physics, B. Cheremushkinskaya str. 25, Moscow, \\ Russia \\ 4 University of Nevada, Department of Physics, Mail Stop 220, Reno, Nevada, USA \\ 5 Osaka University, Graduate School of Engineering, 2-6 Yamada-oka, Suita, Osaka, Japan
}

\begin{abstract}
We have reported the argon plasma waveguide produced in an alumina $\left(\mathrm{Al}_{2} \mathrm{O}_{3}\right)$ capillary discharge and used to guide ultrashort laser pulses at intensities of the order of $10^{16} \mathrm{~W} / \mathrm{cm}^{2}$. A onedimensional magnetohydrodynamic (MHD) code was used to evaluate the average degree of ionization of Ar in the preformed plasma channel. The spectrum of the propagated laser pulse in the Ar plasma waveguide was not modified and was well reproduced by a particle-in-cell (PIC) simulation under initial ion charge state of $\mathrm{Ar}^{3+}$ in the preformed plasma waveguide. The optimum timing for the laser pulse injection was around $150 \mathrm{~ns}$ after initiation of a discharge with a peak current of $200 \mathrm{~A}$.
\end{abstract}

\section{INTRODUCTION}

Ultrashort-pulse high-intensity lasers are used for high-field plasma physics under extreme conditions, but can also yield temporally resolved ultrafast phenomena from a plasma. For femtosecond laser technology generated by a chirped-pulse-amplification (CPA) scheme, the high laser intensity and the high optical field are achieved in a tightly focused beam. In the case of a focused Gaussian beam, the interaction length is approximately a Rayleigh length. To overcome this short interaction length, it is necessary to devise optical waveguides that can be efficiently coupled with an intense laser pulse. For the development of short wavelength light sources, other gases (such as rare-gases) should be used to produce multi-charged-state ions in plasmas. Soft x-ray lasing was observed to be closely correlated to conditions under which the pump laser pulses were guided by gas-filled capillary discharge waveguides. The capillary was filled with a mixture of xenon (Xe) and hydrogen at a discharge current of $105 \mathrm{~A}$ [1]. An argon plasma channel can be used to provide an argon excimer $\left(\mathrm{Ar}_{2} *\right)$ laser at $126 \mathrm{~nm}$ [2], high-order harmonic generation in the sub-keV photon energy region [3], or soft X-ray lasing at $23.3 \mathrm{~nm}$ in an $\mathrm{Ar}^{8+}$ ion column [4] thus providing sources extending from the vacuum ultraviolet (VUV) to the extreme ultraviolet (XUV) spectral regions. In the case of gas-filled capillary soft x-ray lasers, higher charge state ions $\left(\mathrm{Ar}^{8+}-\mathrm{Ar}^{10+}\right)$ should be produced in order to obtain coherent XUV radiation.

\footnotetext{
${ }^{a}$ e-mail: higashi@cc.utsunomiya-u.ac.jp
}

This is an Open Access article distributed under the terms of the Creative Commons Attribution License 2.0, which permits unrestricted use, distribution, and reproduction in any medium, provided the original work is properly cited. 


\section{EPJ Web of Conferences}

In this paper, we report the behaviour of an argon plasma waveguide produced in the capillary discharge and its use to guide ultrashort laser pulses at laser intensities of the order of $10^{16} \mathrm{~W} / \mathrm{cm}^{2}$. A one-dimensional magnetohydrodynamic (MHD) code was used to simulate the plasma behaviour and to evaluate the degree of ionization of Ar in the plasma waveguide. In our experiment the spectrum of the propagated laser pulse in the argon plasma waveguide was not modified, and it was well reproduced by a particle-in-cell (PIC) simulations.

\section{EXPERIMENT AND DISCUSSION}

\subsection{Experimental setup}

A 10-Hz Ti:sapphire laser at a wavelength of $800 \mathrm{~nm}$ produced a maximum energy of about $40 \mathrm{~mJ}$ with a pulse width of $160 \mathrm{fs}$ [full width at half-maximum (FWHM)]. The laser pulse was then focused onto the entrance of a capillary discharge-preformed plasma channel with a focal diameter of about $30 \mu \mathrm{m}$ (FWHM) in vacuum using a lens with a focal length of $15 \mathrm{~cm}$. The focused laser intensity was fixed at $5 \times 10^{16} \mathrm{~W} / \mathrm{cm}^{2}$. The capillary waveguide consisted of a $1-\mathrm{cm}$ long alumina $\left(\mathrm{Al}_{2} \mathrm{O}_{3}\right)$ tube with a diameter of $300 \mu \mathrm{m}$. Argon gas flowed through a gas cell to the pumping vacuum chamber for optical diagnostics, such that the steady-state pressure between the gas cell and the vacuum section was uniform. The pressure in the vacuum chamber was maintained at less than $1 \times 10^{-6}$ Torr. A stainless-steel grounded (earth) electrode was coaxially connected at the entrance of the capillary, with the laser pulse entering the capillary through a tube of diameter $300 \mu \mathrm{m}$. The cathode, also the cupper electrode, was located at the exit of the capillary in a high-vacuum of less than $1 \times 10^{-6}$ Torr. The storage capacitance was $2 \mathrm{nF}$, charged to between 20 and $30 \mathrm{kV}$. The pulse width of the discharge current was 100-150 ns (FWHM) under these conditions. The maximum discharge peak current was about $200 \mathrm{~A}$ at a discharge time delay of about $150 \mathrm{~ns}$. The transmission coefficient of the laser pulse through the plasma channel with the electron plasma density in the range of $(1-2) \times 10^{18} \mathrm{~cm}^{-3}$ was maximized at this discharge time delay. In addition, the visible emission from the plasma jet at the exit of the capillary was also observed by the spectrometer to permit evaluation of the preformed ionization charge state.

\subsection{Argon preformed plasma channel in a capillary}

To compare the experimental results with those from simulations, we used a one-dimensional dissipative MHD code to investigate the plasma dynamics of the argon-filled capillary discharge-produced plasma waveguide. For the numerical simulation, we assumed that the capillary was prefilled with non-ionized argon gas of uniform density and temperature. The discharge was initiated by a pulse of current driven by an external circuit. We used a two-temperature (ion $T_{i}$ and electron $T_{e}$ ) one-fluid MHD approximation, and since the length of the capillary was much larger than its diameter, we also used a one-dimensional (1D) approximation [5].

Figure 1 shows the distributions in the $(r, t)$ plane of electron density (a) in units of $10^{18} \mathrm{~cm}^{-3}$, electron temperature (b) in units of $\mathrm{eV}$, and ion charge state (c). The scales show values of electron density, electron temperature, and ion charge state. The overall picture of the discharge dynamics is as follows: after the breakdown the current pulse heats the plasma and creates an azimuthal component of the magnetic field. Pinching of the plasma is negligibly weak. The plasma is constrained from expansion in the radial direction by the elasticity and inertia of the capillary walls. The simulation showed that the characteristic time for the penetration of an electric field in the plasma, the skin time, was of the order of $1 \mathrm{~ns}$. As a result, the electric field is homogeneous, and consequently the electric current penetrates into the plasma very quickly, and the plasma is heated and ionized locally. During approximately the first $100 \mathrm{~ns}$, the electron density, temperature, and degree of ionization remain radially uniform and grow with time. Then thermal conduction comes into play. The electric field and plasma pressure were found to be radially homogeneous, but the plasma temperature was inhomogeneous since the plasma is cooled 
(a)

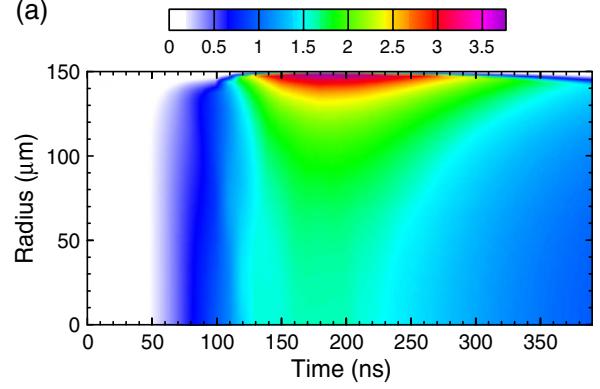

(c)

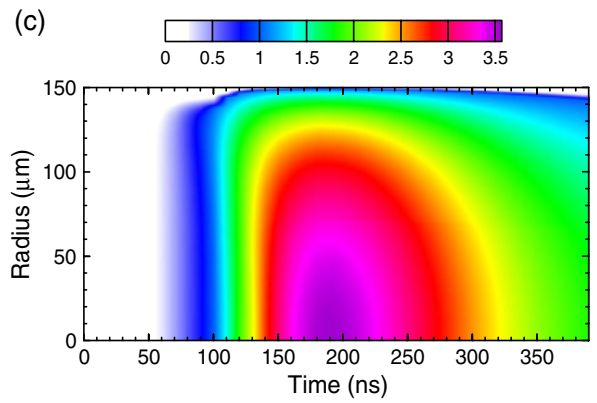

(b)
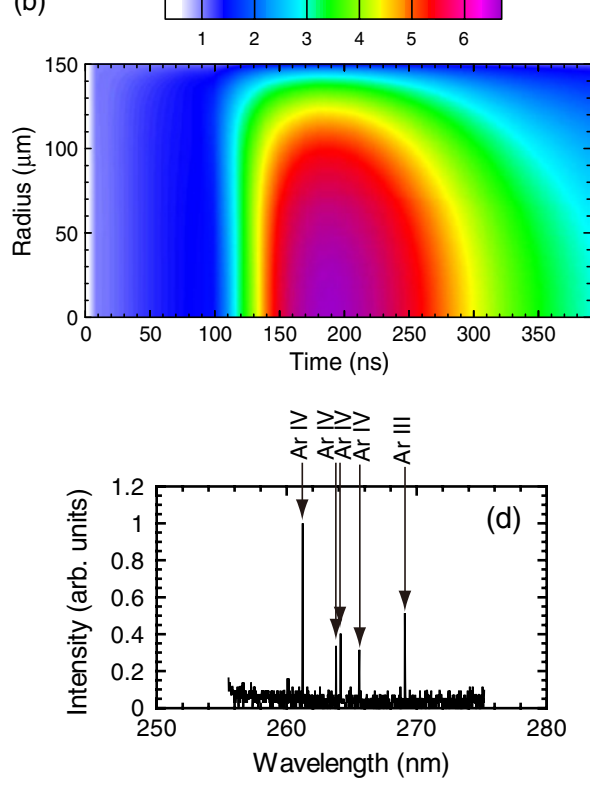

Figure 1. Distributions in the $(r, t)$ plane of electron density (a) its temperature (b), ionic charge state (c), and visible emission spectrum of the preformed argon plasma (d), respectively.

by the capillary wall. As a result, the minimum in the plasma density appears on the axis where the calculated electron density was estimated to be $(1-1.5) \times 10^{18} \mathrm{~cm}^{-3}$. It is noted that the matched spot diameter of $50 \mu \mathrm{m}$ (FWHM), and larger than the focal spot diameter, $30 \mu \mathrm{m}$ (FWHM) at the entrance of the capillary. In this case, the spot diameter would oscillate in the capillary and the measurement of the spot size at the output does not give the matched spot of the guidance [6]. The maximum values of electron density, ion and electron temperatures and the degree of ionization are achieved after the current maximum. Then the plasma is found to be almost immobile in the radial direction. The magnitude of the plasma parameter gradually diminishes with decreasing electric current. The plasma temperature peaks on axis, and this temperature profile produces an axial minimum in the electron density. It was found that the time averaged-ionization charge state was predicted to be $\mathrm{Ar}^{3+}$ resulting in $\mathrm{Ar}$ IV $\left(\mathrm{Ar}^{3+}\right)$ emission which is also in agreement with the measurement, as shown in Fig. 1(d).

\subsection{Laser spectral behaviour}

Figure 2 shows laser spectra recorded in front of the laser window of the vacuum chamber (a) and at the exit (b) of the capillary waveguide. The spectrum of the laser pulse was measured by the scattering from the surface of the laser window. It is necessary to note that before the capillary there is a weak modulation of the laser spectrum at the wavelength of 792 and $795 \mathrm{~nm}$ because of the slight sharp after a laser pulse compressor in the CPA Ti:sapphire laser system due to the insufficient phase compensation. So this spectral modulation was not due to the ionization effect in the capillary plasma. The central wavelength was almost the same in both cases, measured at front of the laser window and at the exit of the capillary. The spectral intensities at 792 and $795 \mathrm{~nm}$ were slightly increased, because Ar ions were additionally ionized from $\mathrm{Ar}^{3+}$ to $\mathrm{Ar}^{8+}-\mathrm{Ar}^{10+}$ by the strong laser field during the laser pulse rise time. The refractive index changed in time, i.e., the blueshift components appeared. However, the change of the refractive index in the preformed plasma was smaller than that in a neutral gas. As a result we observed only slight spectral change. It is noted that the blueshift of $8 \mathrm{~nm}$ was observed without the 

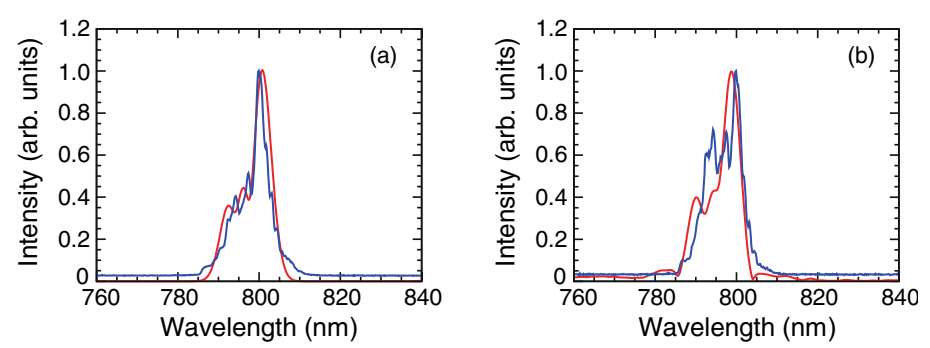

Figure 2. Experimental (blue) and numerical (red) time-integrated spectra of the laser pulse at the entrance (a) and the exit (b) of the capillary with the preformed plasma at a delay time of $150 \mathrm{~ns}$.

preformed plasma channel, i.e., in the neutral gas medium. We reproduced the spectral behaviour of the laser pulse in a plasma waveguide by use of computer PIC code. The averaged ionization state of $\operatorname{argon}$ ions on the laser axis at $r=0$ was $\mathrm{Ar}^{3+}$ at the discharge time of $150 \mathrm{~ns}$, as shown in Fig. 1(c). The red line represents the result of numerical simulation by use of the 1D PIC code (PICLS1D) [7], which supports the presence of additional ionization. We used the one-dimensional PIC code, which takes into account optical-field-induced ionization (OFI), collisions, and recombination in the plasma between several levels of the argon ions. The simulation showed that $\mathrm{Ar}^{10+}$ ions had become the main charge state due to ionization by the laser field in the plasma channel. The presence of this state of ionization $\left(\mathrm{Ar}^{10+}\right)$ was also confirmed by the spectral behaviour simulation. It should be noted that the mean ion charge state would be lower due to the overestimation arising from the 1D simulation. In the case of $2 \mathrm{D}$ or $3 \mathrm{D}$ simulations, the mean electron density would be lower due to the radial ionization and expansion of the plasma, resulting in the lower ion charge state production. It was found that the production of $\mathrm{Ar}^{8+}$ needed for soft x-ray lasers was theoretically expected at laser intensities of the order of $10^{16}-10^{17} \mathrm{~W} / \mathrm{cm}^{2}[4]$.

\section{SUMMARY}

We have investigated guiding of a laser pulse in a capillary discharge-produced argon plasma channel and obtained its spectrum. We found that the average-ionization charge state in the capillary was $\mathrm{Ar}^{3+}$ at a peak discharge current of $200 \mathrm{~A}$. The spectrum of the propagated laser pulse in the argon plasma waveguide was not modified significantly and it was well reproduced in the PIC simulation. The optimum timing of the laser pulse injection was around $150 \mathrm{~ns}$ for the discharge with peak current of $200 \mathrm{~A}$. We showed that the laser propagates in a preformed argon plasma capillary with additional ionization.

\section{References}

[1] A. Butler, A. J. Gonsalves, C. M. McKenna, D. J. Spence, S. M. Hooker, S. Sebban, T. Mocek, I. Bettaibi, and B. Cros, Phys. Rev. Lett. 91, 205001 (2003)

[2] M. Kaku, T. Higashiguchi, S. Kubodera, and W. Sasaki, Phys. Rev. A 68, 023803 (2003)

[3] B. M. Luther, Y. Wang, M. C. Marconi, J. L. A. Chilla, M. A. Larotonda, and J. J. Rocca, Phys. Rev. Lett. 92, 235002 (2004)

[4] M. J. Grout, G. J. Pert, and A. Djaoui, J. Phys. B 31, 197 (1998)

[5] N. A. Bobrova, S. V. Bulanov, D. Farina, R. Pozzoli, T. L. Razinkova, J.-I. Sakai, P. V. Sasorov, and I. V. Sokolov, Laser and Particle Beams 18, 623 (2000)

[6] A. J. Gonsalves, T. P. Rowlands-Rees, B. H. P. Broks, J. J. A. M. van der Mullen, and S. M. Hooker, Phys. Rev. Lett. 98, 025002 (2007)

[7] Y. Sentoku and A. J. Kemp, J. Comp. Phys. 227, 6846 (2008) 